\title{
Morphologic and molecular pathway of cushing syndrome cardiomyopathy
}

\author{
Andrea Frustaci $\mathbb{I}^{1}{ }^{1}$ Claudio Letizia $^{1} \cdot$ Cristina Chimenti $^{1}$
}

Received: 23 May 2017 / Accepted: 1 June 2017 / Published online: 23 June 2017

(C) Springer Science+Business Media, LLC 2017

Dear Sir,

With the present letter we would like to draw your attention to some major statements of the editorial by Vassiliadi DA and Tsagarakis S titled "Cardiac hypertrophy in Cushing's syndrome: if not hypertension then what", published recently in Endocrine (2017; 56:453-455).

Specifically, in their report, the authors declare that the mechanisms of human Cushing cardiomyopathy are still unknown. With this statement they miss two recent publications based on endomyocardial biopsy studies of patients affected by Cushing cardiomyopathy where both histologic and molecular investigations have been obtained $[1,2]$. Here it is demonstrated in a small number of patients (eight patients with Cushing cardiomyopathy, before and after cortisol normalization) that the increased plasma cortisol levels affecting Cushing disease cause, through activation of FOXO (Forkhead box) transcription factor, a 28 times increase of atrogin 1 and ubiquitin. This mechanism promotes proteasome proteolysis and then myofibrillolysis and cell death. Reduction of myofibrillar content of myocardiocytes is, however, a reversible event, as documented by a control biopsy at 1 year from adrenalectomy and normalization of plasma cortisol levels. Indeed, myofibrillolysis cell area reduced from 61 to $22 \%$, and was paralleled by recovery of cardiac dimension and function.

Clinical implications from these observations are that chronic administration of steroids because of hematologic, allergic or autoimmune diseases can be followed by a toxic cardiomyopathy that, if recognized, can be potentially reverted by drug discontinuation.

Acknowledgements This study was funded by AIFA grant 2016003014-28

\section{Compliance with ethical standards}

Conflict of interest The authors declare that they have no competing interests.

Ethical approval All procedures performed in studies involving human participants were in accordance with the ethical standards of the institutional and/or national research committee and with the 1964 Helsinki declaration and its later amendments or comparable ethical standards.

Informed consent Informed consent was obtained from all individual participants included in the study.

\section{References}

1. A. Frustaci, C. Letizia, R. Verardo, C. Grande, C. Calvieri, M.A. Russo, C. Chimenti, Atrogin-1 pathway activation in Cushing syndrome cardiomyopathy. JACC 67, 116-117 (2016)

2. A. Frustaci, C. Letizia, R. Verardo, C. Grande, L. Petramala, M.A. Russo, C. Chimenti, Cushing syndrome cardiomyopathy: clinicopathologic impact of cortisol normalization. Circ. Cardiovasc. Imaging 9(4), e004569 (2016)

\footnotetext{
Andrea Frustaci

biocard@inmi.it

1 La Sapienza University, Rome, Italy
} 